OBETS. Revista de Ciencias Sociales Vol. 9, n. ${ }^{\circ}$ 1, 2014; pp. 15-42

ISSN: $1989-1385$

DOI: 10.14198/OBETS2014.9.1.01

\title{
LA MÚSICA COMO LENGUAJE DE LAS EMOCIONES. UN ANÁLISIS EMPÍRICO DE SU CAPACIDAD PERFORMATIVA
}

\author{
MUSIC AS A LANGUAGE OF EMOTIONS. AN EMPIRICAL \\ ANALYSIS OF ITS PERFORMATIVE CAPACITY
}

\author{
Antonio $\mathrm{F}^{\mathrm{o}}$. Alaminos Fernández \\ University of Nordland, Noruega \\ alam.alaminos@gmail.com
}

\begin{abstract}
Resumen
Esta investigación estudia la relación entre las emociones y la música. Se efectúa un diseño de test-retest donde los individuos expresan qué música escucharían según estados de ánimo, para posteriormente pedir a una segunda muestra, distinta a la anterior, que valore en qué grado considera que dichas canciones expresan esos mismos estados de ánimo. Se ha efectuado un análisis de los rasgos musicales de dichas canciones. Se concluye que tanto en las emociones como en los rasgos musicales existe una dimensionalidad subyacente. En el caso de las emociones corresponde con las propuestas del diferencial semántico. En los rasgos musicales, se determinan dos dimensiones aún sin etiquetar teóricamente. Finalmente, se muestra la relación empírica entre rasgos musicales y emociones mediante un modelo estructural de medición.

Palabras clave: Música, Emociones, Diferencial Semántico, Modelo Estructural.

\section{Abstract}

This research studies the relationship between emotions and music, with a test-retest design. First, individuals express which music they listen according to their moods, and later a second sample, independent of the previous one, are asked in which grade considers that these songs express the same mood. After completed an analysis of the musical features, we conclude that both emotions, and musical features, show an underlying dimensionality. In the case of emotions, corresponds with the proposal of the semantic differential. In musical terms, two dimensions are empirically determined. Finally, the empirical relationship between musical features and emotions shown, are tested with a structural model.
\end{abstract}

Keywords: Music, Emotions, Semantic Differential, Structural model. 


\section{INTRODUCCIÓN}

Que la música es un lenguaje constituye prácticamente un tópico de general aceptación. Incluso determinadas frases comunes apuntan a una eficacia pragmática, real o imaginaria, especialmente dirigida a las emociones. Un ejemplo de ello, la frase "la música amansa las fieras", destaca precisamente de forma extrema dicha finalidad. Sin embargo, no es una frase casual, en la medida que varias investigaciones recientes (Juslin P.N. and Sloboda J. A., 2010; Menon, V. \& D.J. Levitin, 2005; Krumhansl, 1997; Blood, A.J., R.J. Zatorre, P. Bermúdez \& Evans, A.C. 1999; Brown, S., Martínez M. \& Parsons, L.M., 2004; Blood, A. \& R.J. Zatorre, 2001;) proponen que la música es percibida por las áreas del cerebro que reciben estímulos de carácter emocional, asociadas a sentimientos. Para sentir la música y experimentar sus consecuencias no es preciso que intervengan de forma explícita las capacidades reflexivas de los individuos. (Koelsch, S., T.C. Gunter, A.D. Friederici \& Schröger, E., 2000). Esta comunicación directa a las emociones y los sentimientos que establece la música le concede una capacidad equivalente a la poesía, si bien entre individuos sin una cualificación cognitiva o sensibilidad especialmente cultivada. (Meyer, L.B., 1956). La música como lenguaje, (Levitin, D.J. \& V. Menon, 2003) le habla incluso a los que no están prestando atención. Estas cualidades emocionales ya están expuestas desde sus inicios en la filosofía occidental. Así, Aristóteles señala como

...en los ritmos y las melodías que encontramos las semejanzas más perfectas en consonancia con su verdadera naturaleza de la ira y la mansedumbre, de la fortaleza y la templanza, como también de sus contrarios y de todas las otras disposiciones morales... los ritmos, unos tienen un carácter más reposado, otros más movido, $y$ de estos unos inducen emociones más vulgares, $y$ otros otras emociones más propias de un hombre libre. (Aristóteles, 1982: 306).

En la reflexión sobre los sonidos, se incluían tanto los sonidos armónicos (música) como los sonidos y ruidos propios de la naturaleza, capaces de evocar estados de ánimo. El sonido de la lluvia, de una cascada de agua o el canto mismo de los pájaros, generan estados de ánimo, en ocasiones imitados por los instrumentos musicales.

La música, y los sonidos en general, se procesan en varias zonas del cerebro y no solamente en las responsables del lenguaje y el sonido. En los estudios efectuados mediante tomografía por emisión de positrones (PET/TC) se aprecia que áreas relacionadas con otros sentidos, como el de la visión, se activan en relación a estímulos musicales. (Koelsch, S. \& Friederici, A.D., 2003). Las evidencias de actividad cerebral muestran como la memoria o la evocación se acti- 
van con la música. En ese sentido, la música ofrece una vía paralela que puede reforzar, modificar e incluso cambiar en su opuesto mensajes verbales o visuales.

Esto es especialmente importante en determinadas áreas. Así, por ejemplo, la música en comunicación o publicidad (North, A. and Hargreaves, D.J., 2010) y su anclaje cultural como lenguaje, la "afinación" conceptual cuando aparece asociado a mensajes y recomendaciones publicitarias o su capacidad para evocar emociones o situaciones. Es evidente que la relación entre música y emociones o afectos es tan intensa que actúa bidirecionalmente. Así, la conexión entre música y emociones ha sido también investigada en sentido contrario como se muestra en varios estudios (Konecni, 2010: 714). En ese sentido, la primera fase de esta investigación explora como los estados emocionales de los individuos pueden sugerir melodías y canciones. Es algo equivalente a cuando un individuo elige una canción para escuchar según su estado de ánimo. En el sentido opuesto, el estado de ánimo de un individuo puede cambiar según las músicas que le rodean.

El estudio de la relación de la música con los sentimientos y los comportamientos ha interesado a múltiples disciplinas que triangulando han confirmado dicha eficacia. Especialmente destaca el enfoque neurofisiológico (Koernst y Siebel, 2005; Zatorre, Chen \& Penhune, 2007), los procesos cognitivos y psicológicos (Konecni, 2010), complementados con la antropología (Hannon and Trainor, 2007), o la sociología (Santacreu, 2002). Algunos autores (Fritz, Jentschke, Gosselin, Sammler, Peretz, Turner, Friederici and Koelsch, 2009) han postulado que la música es un lenguaje universal, produciendo al menos tres emociones que son reconocibles en cualquier cultura. Estos estudios se encuentran en fase muy temprana y no existen evidencias suficientes sobre la universalidad de las emociones generadas por la música. En ese sentido, el estudio de la música y sus efectos sociales y emocionales es un área de actividad cada vez más prometedora, como consecuencia de los procesos de triangulación que permiten las diferentes disciplinas.

\section{OBJETIVOS Y ANTECEDENTES}

Existe un objetivo básico que enmarca diferentes subhipótesis. La hipótesis es establecer primero, y detallar después, la asociación entre música, emociones y sentimientos, mediante un enfoque que integre las propiedades formales de los lenguajes musicales con la medición de emociones. ¿Es factible agrupar empíricamente las diferentes cualidades musicales en función a los perfiles (dimensiones) que se aprecien en las canciones consideradas? ¿Se puede observar relación entre estos rasgos y las emociones asociadas a las canciones analizadas? ¿Responden las emociones a una estructura dimensional latente? ¿Qué relación 
mantiene con las propuestas de diferencial semántico? Estas son algunas de las preguntas que desarrollaremos en este estudio. Las conexiones entre música (como lenguaje) y emociones, sentimientos e incluso contenidos semánticos se han investigado previamente preferentemente desde varios campos: la musicología, la neurología y la psicoacústica, la psicología, la sociología y en menor medida el marketing y comunicación. En muchos sentidos, el estudio unidisciplinario de la relación entre música y las emociones deja en evidencia importantes lagunas, así como una falta de intercambio de información que permita validar, por triangulación teórica, muchas de las conclusiones provisionales que se van alcanzando.

Así, considerando los diseños de investigación podemos encontrar desde los más "físicos" hasta los más "psicológicos". Los procedimientos metodológicos empleados incluyen el reconocimiento de las áreas cerebrales que se activan con la música, donde existe una evidencia empírica de activación, pero no del significado real de dicha activación. Como señala Koelsch

So far, the majority of neuroscience studies on human emotion have used static visual images as experimental stimuli. However, during the past years, the neurosciences have discovered that music is also a valuable tool to investigate emotion. Important advantages of music are (1) that music is capable of inducing emotions with a fairly strong intensity, (2) that such emotions can usually be induced quite consistently across subjects, and (3) that music can induce not only unpleasant, but also pleasant emotions (which are rather difficult to induce by static images). Neuroscience studies on the investigation of emotion with music basically indicate that networks of limbic and paralimbic structures (such as amygdala, hippocampus, parahippocampal gyrus, insula, temporal poles, ventral striatum, orbitofrontal cortex, and cingulate cortex) are involved in the emotional processing of music. These structures have previously been implicated in emotion, but the functional significance of each of these structures is still not well understood. (Koelsch, 2005a: 1)

Con posterioridad, los desarrollos de esa línea de investigación han consolidado las relaciones empíricas entre la activación de áreas cerebrales y estímulos musicales. (Koelsh, 2005; Koelsh, 2010). Otras estrategias complementarias desde la neurología han considerado junto a las resonancias magnéticas, el nivel de oxígeno en sangre asociado a los estados de ánimo inducidos por diferentes tipos de música (Mitterschiffthaler, M. Fu, C, Dalton, J. Andrew, C.M. y Williams S.; 2007). En este diseño de estudio, el equipo de investigación proponía la clasificación de los tipos de música ( 5 tristes, 5 alegres y 10 neutros). Sin embargo, estos tipos de músicas no habían sido determinados mediante investigación empírica, según las opiniones de la sociedad. Tanto las músicas 
como sus significados teóricos eran propuestas del equipo de investigación. En ese sentido, tanto los tipos de emociones como la clasificación de las canciones fueron establecidas a priori por los investigadores y tras un debate teórico. No fueron establecidos según la subjetividad de los individuos y, menos aun internamente por los sujetos experimentales. Los estados de ánimo se midieron mediante un breve cuestionario. Sin embargo, la vinculación sustantiva entre emociones y lenguaje musical solo puede aportarse desde otras disciplinas, que permitan conocer las estructuras y los rasgos musicales, mediante diseños por los que pueda demostrarse una relación explícita consistente y válida. El nivel de oxígeno en sangre según la canción escuchada no es, tomado aisladamente, suficientemente concluyente respecto a la inducción de emociones.

No obstante, las aportaciones actuales son profundamente prometedoras, en la medida en que "Recent demonstrations that music is capable of conveying semantically meaningful information has raised several questions as to what the underlying mechanisms of establishing meaning in music are, and if the meaning of music is represented in comparable fashion to language meaning". (Steinbeis N, Koelsch S., 2008). Sin embargo, son muy escasos los intentos de formalizar una relación entre los rasgos distintivos de la música (en tanto que lenguaje musical) y sus efectos emocionales. Esta aproximación exige al menos una elevada competencia musical y el análisis de las preferencias subjetivas de los individuos según sus estado emocional. Por sí mismas, la activación de áreas vinculadas a lo visual o la memoria solo pueden mostrar que puede existir alguna asociación, pero nada concluyente sobre la naturaleza de dicha relación.

Desde la psicología han sido varios los intentos de medición y desarrollo de estrategias para conocer como la música genera sentimientos. (Zentner, M.; Grandjean, D.; Scherer, K., 2008). En ese sentido, la Geneva Emotional Music Scale es un intento interesante de asociar estados de ánimo con música. Una aproximación semejante es la de Vieillard et alt. (2008) que efectúa un contraste entre músicas predefinidas y sujetos experimentales. Sin embargo, el diseño es claramente mejorable. Así, en lugar de ofrecer a los sujetos una serie de músicas clasificadas previamente por el investigador según las emociones que cree debería despertar (clasificación establecida por el investigador), se debería dejar libertad al individuo para que, personalmente, indique las canciones que prefiere escuchar según estado de ánimo. Esa fase exploratoria, permite una clasificación y estudio posterior con un mayor grado de validez. Las emociones que finalmente se consideran en esta investigación han sido elegidas como expresión de los pares de antónimos definitorios de las dimensiones del diferencial semántico. En los escalamientos mediante diferencial semántico, los pares de atribu- 
tos o cualidades son especificas para el concepto medido. No existe un escalamiento tipo, que pueda ser empleado universalmente para cualquier concepto.

\section{EL DISEÑO Y LOS DATOS}

Para testar la hipótesis sobre la relación entre música y emociones se han empleado datos primarios, recolectados para esta investigación mediante el uso de nuevas tecnologías de la comunicación, esencialmente facebook y tuenti. Para ello, se ha tomado como muestra a jóvenes entre 15 y 24 años. Una parte de ellos participó en el establecimiento de la relación entre estados de ánimo y su influencia en la selección de canciones para escuchar en determinados momentos. Un grupo de jóvenes diferentes participó en el establecimiento del recorrido empírico contrario: determinar que emociones provocaba la audición de dichas canciones. De esta forma, el nexo entre tipo de emoción y sus canciones correspondientes quedaban validadas en los dos sentidos. De la emoción a la canción que se prefiere escuchar y de la canción a la emoción que provoca.

El diseño parte de la idea según la cual, un grupo de entrevistados decide libremente que canciones escucharía en función de sus estados de ánimo. Posteriormente, a una segunda muestra, se le pide que valoren en qué grado cree que cada canción puede expresar un estado de ánimo. Las dos muestras son independientes entre sí, y la segunda actúa para determinar la consistencia entre canción y estado de ánimo. De esta forma se define la primera matriz de datos, que recoge la asociación libre por parte del entrevistado entre estado de ánimo y canción. En una segunda fase, otros entrevistados valoran de cero a diez cada canción según creen que pueden expresar estados de ánimo. En resumen, el diseño de la recolección de datos se desarrolla en dos fases en la investigación ha seguido el siguiente proceso:

Fase I. Exploratoria

1. Información subjetiva espontánea sobre las canciones que se escuchan según estado de ánimo. (Ocho estados de ánimo). Muestra de jóvenes.

2. Análisis musical de las canciones.

3. Análisis estadístico de los rasgos musicales.

4. Selección de canciones típicas. (Ocho canciones típicas, una para cada estado de ánimo)

Fase II. Validación

1. Encuesta preguntando a los entrevistados que concedan una puntuación de cero a diez a cada una de las ocho canciones, para cada uno de los sentimientos considerados. 
El análisis está definido para determinar la relación entre estados de ánimo y los rasgos musicales de las canciones. Para ello, el primer análisis se orienta a determinar la dimensionalidad subyacente a las emociones consideradas. Este análisis de dimensionalidad permite apreciar la presencia de estructuras latentes que den cuenta de la variabilidad de las emociones. Como era esperado, varias de ellas están asociadas entre sí, mostrando covariación. Tras el análisis de dimensionalidad de las emociones, según el modelo de diferencial semántico de Osgood, Sucy y Tannenbaum, se procede al análisis de los rasgos musicales de las canciones. Las canciones han sido analizadas de acuerdo a criterios musicales, determinando sus propiedades y en qué forma son diferentes entre ellas, generando una muestra de canciones y sus características. Se ha efectuado un análisis de escalamiento óptimo, mediante mínimos cuadrados (ALS), determinando la posible existencia de dimensiones latentes tras la agrupación de los efectos causados por los rasgos musicales. En resumen, el primer análisis analiza la relación que las emociones establecen entre ellas. El segundo, la relación entre los rasgos musicales, según las emociones que expresan las canciones.

Fase III. Análisis.

2. Análisis dimensional mediante análisis factorial. Tres dimensiones: evaluación, potencia y actividad confirmando las propuestas de Osgood, Sucy y Tannenbaum sobre "orientación psicológica hacia objetos" (escala de diferencial semántico).

3. Análisis dimensional (mediante escalamiento óptimo ALS) de los rasgos musicales de las canciones estudiadas y escalamiento óptimo de los rasgos musicales estudiados.

Por último, se especifica un modelo estructural relacionando la estructura psicológica (dimensiones: evaluación, potencia y actividad) y la estructura dimensional del lenguaje musical. Este modelo estructural indicaría tanto la dimensionalidad como las relaciones entre ellas, en los dos planos del lenguaje musical (rasgos) y las emociones expresadas mediante diferencial semántico.

Fase IV. Modelo integrado: la conexión empírica del lenguaje musical con los sentimientos.

En términos de datos, la investigación se apoya sobre tres matrices de datos complementarias. Por un lado la matriz de datos que asocia estado de ánimo y canción (en ese orden), por otro la matriz de datos que relaciona canciones con estados de ánimo. Por último, una tercera matriz de datos dónde se incluye los resultados del análisis de los rasgos musicales de las canciones. 
Las dos muestras (exploratoria y de validación) se han obtenido mediante un sistema de "bola de nieve" corregido. Este sistema de muestreo de bola de nieve puede presentar sesgos en la medida que se recorran grupos homogéneos en gustos o preferencias. Para incrementar la diversidad de la muestra, el diseño dispersa la muestra mediante el empleo de conexiones de segundo orden (conocido de un conocido) donde no se comparten los contactos en las diferentes subredes. Es decir, se entrevista al conocido de un conocido que no conoce a los conocidos de primer orden. La inclusión en la muestra exige la no pertenencia a las subredes de contactos de primer orden, produciéndose un diseño que se expande irradiando los contactos en diferentes niveles desde la red interior hacia el exterior. Se intenta garantizar una mayor heterogeneidad entre los participantes, reduciendo el riesgo de redundancia en las opiniones y preferencias dentro de una única red de amigos Con ello podemos considerar que la muestra alcanza una elevada diversidad dentro del segmento juvenil estudiado. Las variables de control han sido género, dispersión geográfica de ámbito nacional, nivel educativo y ocupación del joven.

Figura 1. Muestreo mediante "bola de nieve" de segundo orden

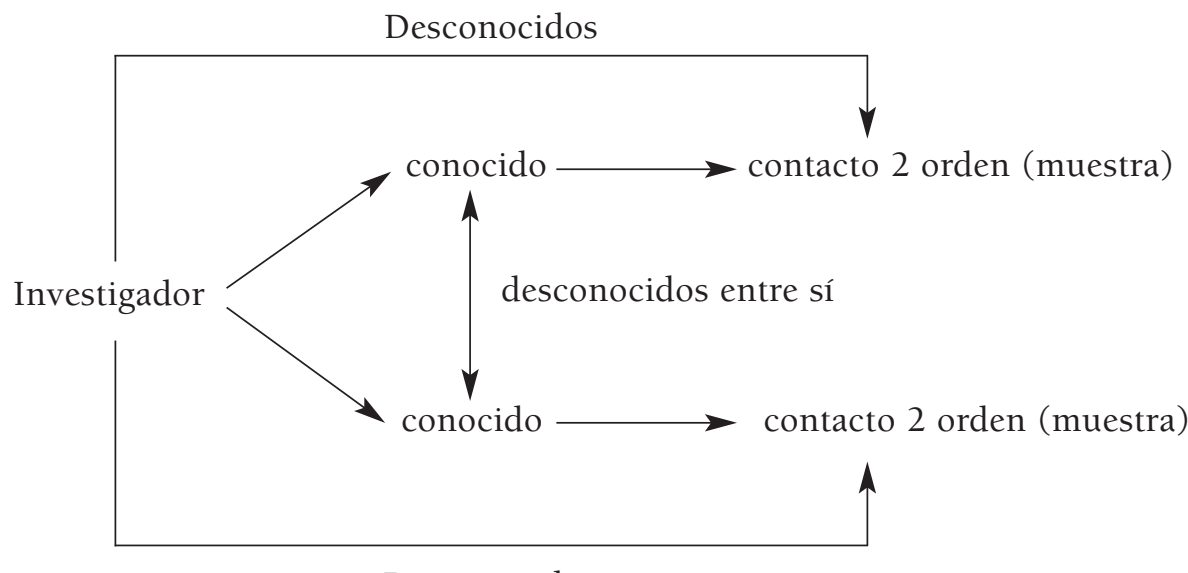

Desconocidos

El diseño muestral ha funcionado de forma óptima, con un nivel de no respuesta total menor del $4 \%$ de los contactos iniciados en la primera muestra (exploratoria, con asociación libre entre canciones y estados de ánimo) y del $14 \%$ en la segunda (donde el entrevistado debía asociar canciones predefinidas y estados de ánimo). Los motivos para no responder no muestran asociación con el tema sobre el que se preguntaba. Como indicamos, se construye una tercera base de datos, con las codificaciones de los rasgos musicales contenidos en cada 
una de las canciones analizadas. A efectos de análisis, esta base de datos fue integrada, en la matriz de datos de la segunda muestra (validación).

Así, la primera muestra con carácter exploratorio para determinar el conjunto de canciones asociadas a estados emocionales tiene un tamaño de 20 casos (entrevistados) y 160 respuestas (canciones). Su composición de género es del $47 \%$ hombres y $53 \%$ mujeres, con edades comprendidas entre los 15 y los 24 años, con dispersión geográfica nacional y un 64\% estudiando y el 28\% desempleados y el $8 \%$ trabajando. El planteamiento para la recogida de datos fue formal y dinámico. Los entrevistados debían autocumplimentar la información que se les solicitaba y consultar cualquier duda en línea. Se les administró el siguiente formulario de recogida de datos.

Formulario:

Presentación de la recogida de datos. Con carácter informal.

Si te encuentras en los siguientes estados de ánimo, ¿que música prefieres escuchar?

Lista de estados de ánimo.

Feliz: canción

Con ganas de bailar: canción

Triste: canción

Relajante: canción

Animada: canción

Romántica: canción

Nostálgica: canción

Tensión: canción

La información sobre categorías emocionales y canciones se complementó con información personal del entrevistado sobre conocimiento de idiomas, género, ocupación, lugar de residencia y edad.

Las canciones mencionadas con más frecuencia asociadas a estados de ánimo entraron a formar parte de la segunda fase de investigación. Estas canciones constituyen una submuestra representativa que serán objeto de valoración por la segunda muestra de entrevistados. En el apartado metodológico se amplía la información sobre las propuestas de rasgos distintivos de emociones en la música.

A la muestra de validación se les presentó la selección de ocho canciones y se solicitó que valoraran cada una de ellas de acuerdo a las emociones expresadas en formato de diferencial semántico: Felicidad, Relajante, Animada, Romántica, Nostálgica, Bailable, Triste y Tensión. Junto a las canciones se incorporó el link a Youtube para que se pudiesen escuchar las canciones, en el caso 
de no ser conocidas por el entrevistado. Se obtuvo una muestra de 30 entrevistados, habitual en los estudios psicométricos y neurofisiológicos que investigan los efectos de la música.

Canciones tipo de las categorías emocionales

Moves like Jagger (Maroon 5)

http://www.youtube.com/watch?v=iEPTlhBmwRg

Mad World (Gary Jules)

http://www.youtube.com/watch?v=4N3N1MlvVc4

Black or White (Michael Jackson)

http://www.youtube.com/watch?v=0eShfZKEe_Y\&feature=related

You'r beautiful (James Blunt)

http://www.youtube.com/watch?v=oofSnsGkops

The reason (Hoobastank)

http://www.youtube.com/watch?v=fV4DiAyExN0

Party Rock (LMFAO)

http://www.youtube.com/watch?v=KQ6zr6kCPj8

Con las ganas (Zahara)

http://www.youtube.com/watch?v=yTwzhCKMA7k

Roulette (Zombie Kids)

http://www.youtube.com/watch?v=qTbrb1Jp4wE

A partir de esta información se construye la matriz de datos, con las variables de estado de ánimo, resultado de valorar cada canción de cero a diez, según opinaran los entrevistados en la muestra de validación. A esta matriz de datos se agregan las variables con la codificación de análisis musical y estado de ánimo. Es decir, a la segunda base de datos, donde se recoge las puntuaciones de los entrevistados, se le fusiona los rasgos musicales de las canciones en examen. Así, tenemos una matriz de datos con dos tipos de variable: unas de valoración subjetiva de los entrevistados de las canciones evaluadas y otra con los rasgos objetivos que definen cada canción musicalmente. Esto permite establecer la matriz de covarianzas entre la valoración subjetiva (sentimientos) de los entrevistados y las propiedades musicales de las canciones.

\section{EL ANÁLISIS MUSICAL}

El diseño de la investigación es por sí novedoso en este campo de investigación. Triangula entre las perspectivas Emic y Etic mediante un diseño en tres fases. La primera es de naturaleza exploratoria y emplea el catálogo de emociones pro- 
puesto por varios autores (Santacreu, 2002) para que los sujetos indiquen de forma libre las canciones que asocia a varios estados de ánimo. Como es bien conocido, una de las características principales de la música es el sonido. El sonido tiene cuatro cualidades subjetivas. Estas son Intensidad, Tono, Timbre y Duración. Todas ellas disponen de una explicación física que desarrolla, especialmente, la psicoacústica. Un área de investigación muy próxima a la que nos ocupa pero que por cuestión de parsimonia no ampliamos aquí. Las propiedades del lenguaje que hemos considerado son propias del análisis musical. (Seguí, 1991: 63).

\title{
Cuadro 1. Cualidades del sonido
}

\begin{tabular}{l|l}
\hline Intensidad & Volumen: Fuertes / Débiles \\
\hline Tono & Instrumentación: Agudo / Grave \\
\hline Timbre & Voz: Masculina / Femenina \\
\hline Duración & Tiempo: Largo / Corto \\
\hline
\end{tabular}

Partiendo de Rowell (1983, 156-174), disponemos de una larga clasificación de valores de la música como son los valores tonales, valores textuales, valores dinámicos, valores temporales, y valores estructurales.

\author{
Cuadro 2. Valores Tonales \\ El silencio \\ El tono (Tonalidad) \\ El acorde \\ El color armónico \\ El timbre \\ Cuadro 3. Valores textuales \\ Simple/Complejo \\ Suave/Áspero \\ Delgado/Denso \\ Economía/Saturación \\ Dirección Vertical/Horizontal \\ Centro/Interjuego(Llamar la atención) \\ Confusión \\ Figuración
}

\section{Cuadro 4. Valores Dinámicos}

Cambios de tiempo

Cadencias (Clímax) 


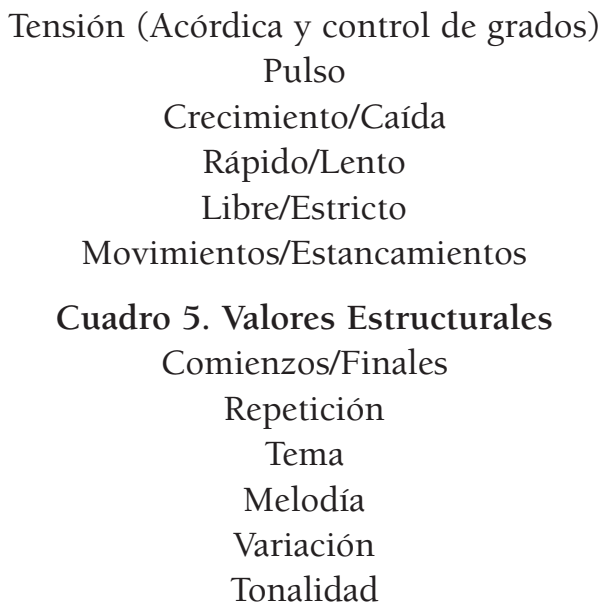

Los valores temporales se pueden integrar dentro de los valores dinámicos. (Santacreu, O. 2002: 111). Partiendo de esta clasificación de cualidades y valores, se utilizaran en el análisis aquellas que muestran mayor capacidad discriminante. De los valores utilizaremos "textura" (servirá para diferenciar entre compleja y simple), "pulso" (para decir si es estricto o libre) y "tonalidad" (la cual indicará rasgos muy básicos considerado mayor o menor). Estas las clasificaremos dentro de las cualidades del sonido. Dentro de la cualidad "Intensidad" encontramos volumen que hace regencia a sonidos fuertes y sonidos débiles, tensión que intenta localizar si la canción tiene o no tensión. En la referida al "Tono" encontramos Tonalidad siendo mayor, menor o las dos en el caso de no sobresalir una de ellas, Instrumentación haciendo referencia a el bloque sonoro de instrumentos de la canción divididos en agudo y grave y como último Textura siendo posible una textura simple o compleja. Seguidamente en la cualidad "Timbre" localizamos la Voz siendo posible la masculina, femenina o el uso de las dos. Finalmente, la última cualidad de duración identifica el Tiempo que puede ser rápido, lento o los dos y Pulso, siendo posible estricto o libre. De esta forma las variables para medir las propiedades del sonido, y que van a ser empleadas para el análisis de las canciones las vemos en el Cuadro 6 (pág. 27).

En la determinación de los rasgos musicales de las canciones propuestas por los entrevistados se han aplicado los anteriores criterios analíticos.

\section{ANÁLISIS}

Vamos en primer lugar a determinar la presencia de dimensionalidad en las emociones que hemos evaluado. Esta dimensionalidad indicaría que las emociones vienen preestructuradas sobre la base de variables latentes. Un referente 


\section{Cuadro 6. Modelo de variables del sonido aplicadas a la música}

\begin{tabular}{l|l}
\hline Intensidad & $\begin{array}{l}\text { Referida al volumen: } 1 \text { - Fuertes / } 0 \text { - Débiles } \\
\text { Tensión: 1- Sí / } 0 \text { - No }\end{array}$ \\
\hline Tono & Tonalidad: 1- Mayor / 0 - Menor / 2 - las dos \\
& Instrumentación: 1 - Agudo / 0 - Grave \\
& Textura:1 - Simple / 0 - Complejo \\
\hline Timbre & Voz: 1 - Masculina / 0 - Femenina / 2 - Las dos \\
\hline Duración & Tiempo: 1 - Rápido / 0 - Lento / 2 - los dos \\
& Pulso: 1 - Libre / 0 - Estricto \\
\hline
\end{tabular}

Fuente: elaboración propia a partir de las clasificaciones anteriores

importante es la propuesta de semantización que se efectúa desde la teoría del diferencial semántico, por el que la imagen de un concepto se organiza en tres dimensiones: Evaluación, Potencia y Actividad. Posteriormente, se efectuará otro análisis de dimensionalidad sobre los datos que expresan los rasgos musicales de las canciones consideradas. En este caso, no existen precedentes teóricos que postulen una organización u otra de las características musicales a efectos de producir emociones. Finalmente, se testa un modelo estructural de medición, relacionando las emociones identificadas en las canciones y los rasgos determinados por el análisis musical.

\subsection{La dimensionalidad de las emociones}

En primer lugar, hemos considerado diagnosticar en que forma los estados de ánimo expresados por las canciones mantienen una afinidad entre sí. En definitiva, determinar la presencia de dependencia o independencia entre ellos. Para analizar la posible dimensionalidad presente en las valoraciones de las emociones que expresan las canciones, y a partir de los datos recogidos en la muestra de validación, se efectúa en primer lugar un análisis de segmentación jerárquica, para averiguar la proximidad en los perfiles de evaluación (cero a diez).

Podemos apreciar la existencia de tres cluster. En primer lugar, se agrupan los sentimientos Animada, Bailable y Feliz. Es importante apreciar como la opción Feliz ha sido interpretada en un sentido de dinamismo. Un segundo grupo lo define la Tensión, que se agrupa tardíamente, y el tercero viene definido por las emociones Nostalgia, Relajante, Tristeza y Romántica. El análisis de conglomerados muestra la presencia de tres segmentos valorativos que apuntan a una dimensionalidad en las variables consideradas. 
Gráfico 1. Conglomerados de emociones Dendrograma que utiliza una vinculación media (entre grupos)

Combinación de conglomerados de distancia re-escalados

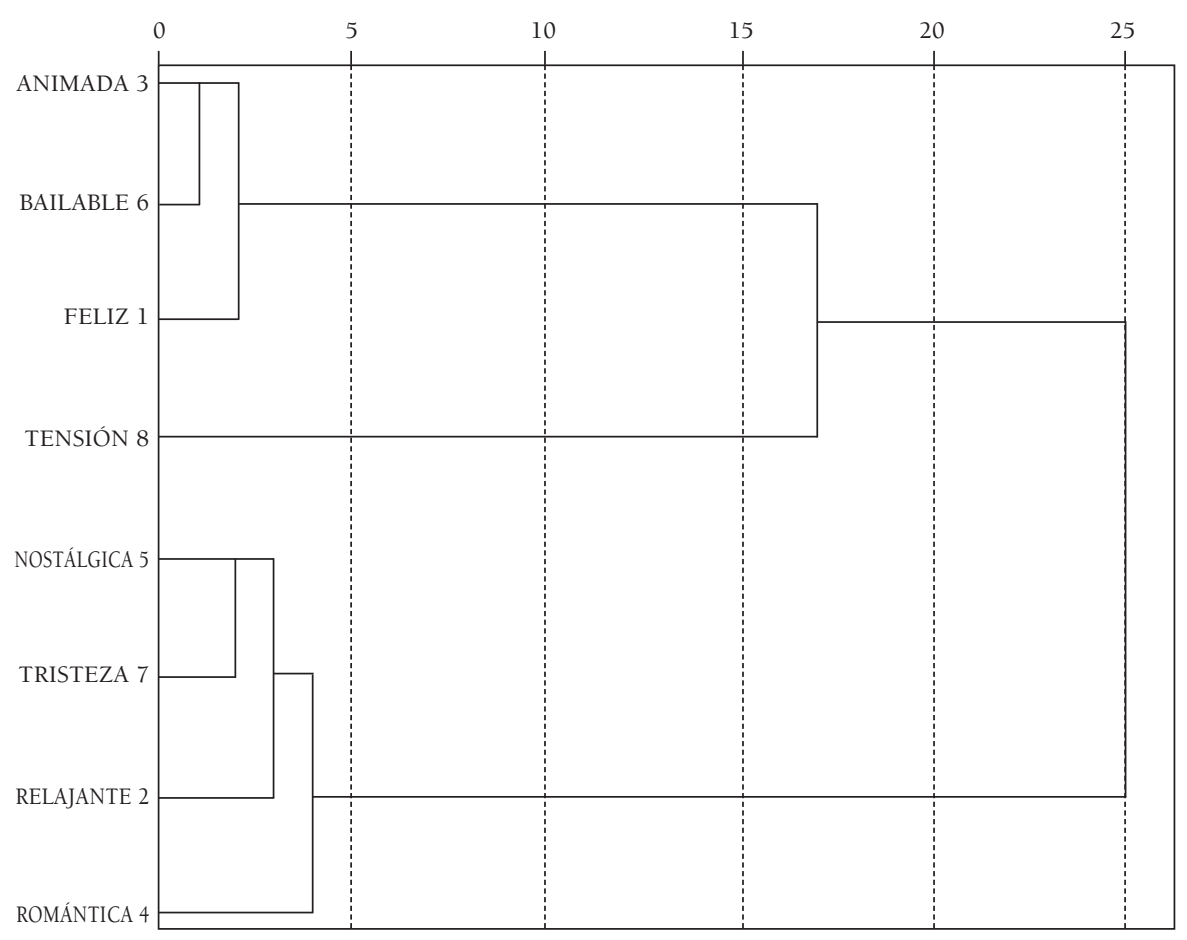

Fuente: elaboración propia sobre datos de la muestra de validación

Esta idea se confirma mediante el análisis factorial de las emociones que expresan cada canción. El análisis factorial determina la presencia de tres dimensiones. Estas dimensiones agrupan las variables consistentemente con la estructura que apreciábamos anteriormente, en el análisis de segmentación jerárquica. Metodológicamente es muy importante la triangulación mediante diferentes métodos de análisis. Esto permite comprobar la consistencia, en la medida que diferentes procedimientos concluyan una misma realidad empírica. Así se evita que se generen estructuras explicativas consecuencia del método empleado. La estructura factorial de tres dimensiones explica el $86 \%$ de la varianza en las valoraciones. Un porcentaje bastante elevado. De la inspección de los coeficientes o cargas factoriales de las diferentes variables en cada factor, mostradas en la siguiente tabla, podemos observar como la agrupación responde a las tres dimensiones propias del diferencial semántico: Evaluación, Potencia y Actividad. 


\section{Tabla 1. Cargas factoriales de las emociones}

Matriz de componentes rotados ${ }^{\mathrm{a}}$

\begin{tabular}{l|c|c|c}
\hline & \multicolumn{3}{|c}{ Componentes } \\
\cline { 2 - 4 } & 1 & $\begin{array}{c}2 \\
\text { (Evaluación) }\end{array}$ & $\begin{array}{c}3 \\
\text { (Potencia) }\end{array}$ \\
\hline ROMÁNTICA &, 880 &,- 180 &,- 157 \\
\hline NOSTÁLGICA &, 826 &,- 380 &,- 302 \\
\hline RELAJANTE &, 762 &,- 319 &,- 110 \\
\hline TRISTEZA &, 731 &,- 536 &, 972 \\
\hline FELIZ &,- 171 &, 915 & \\
\hline ANIMADA &,- 417 &, 854 & \\
\hline BAILABLE &,- 418 &, 823 & \\
\hline TENSIÓN &,- 172 & & \\
\hline
\end{tabular}

Método de extracción: Análisis de componentes principales.

Método de rotación: Normalización Varimax con Kaiser.

a. La rotación ha convergido en 5 iteraciones.

Fuente: elaboración propia sobre datos de la muestra de validación

El gráfico muestra claramente la agrupación dimensional de las emociones consideradas, en las variables latentes de evaluación, potencia y actividad (ver gráfico 2, pág. 30).

La estructura dimensional queda claramente expresada en el gráfico 3. Esta corresponde con un modelo estructural de medición de variables latentes, donde se identifican los conceptos teoricos clave así como la carga explicativa de las diferentes emociones en análisis. La capacidad que muestra cada una de las dimensiones en los diferentes grupos de variables es muy elevada, con cargas medias entre el .73 y el .97 . Se confirma que las emociones atribuidas espontáneamente a las canciones en la primera muestra de individuos, son validadas por la segunda muestra de individuos. Mostrando, así mismo una estructura dimensional que corresponde con las propuestas teóricas de Osgod, Sucy y Tannembaun sobre la semantización.

En ese sentido, a partir de los resultados podemos concluir que las emociones que se asocian a las canciones consideradas muestran una estructura latente, acorde con las propuestas teóricas del diferencial semántico. 
Gráfico 2

Gráfico de componentes en espacio rotado

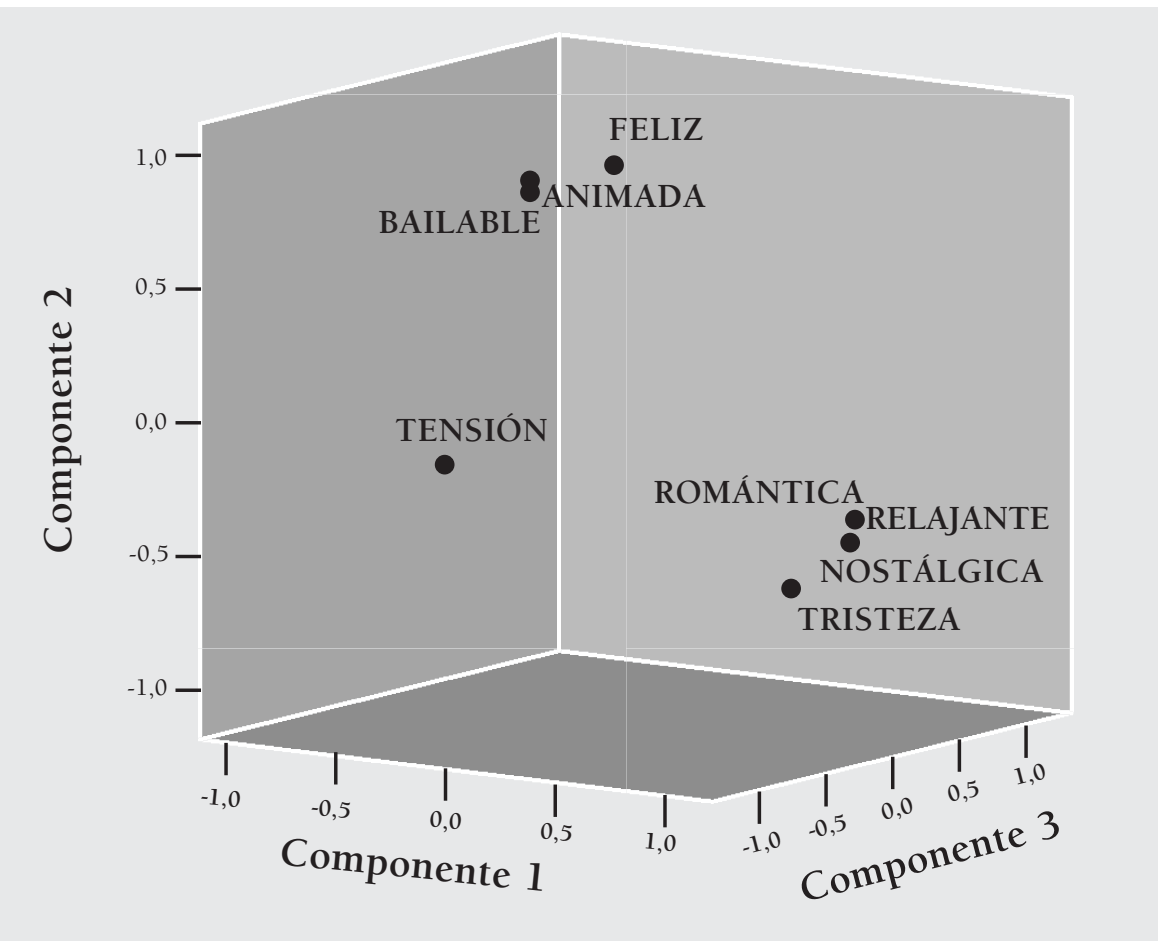

Fuente: elaboración propia sobre datos de la muestra de validación

En un segundo análisis exploramos la estructura en las cualidades musicales consideradas. Con ello se pretende responder a varias cuestiones. ¿Es factible agrupar empíricamente las diferentes cualidades musicales en función a los perfiles (dimensiones) que se aprecien en las canciones consideradas? ¿Se puede observar relación entre estos rasgos y las emociones asociadas a las canciones analizadas? Para poder responder a estas preguntas, es preciso efectuar en primer lugar una transformación empírica de los valores atribuidos a las cualidades musicales.

\subsection{Escalamiento óptimo de los valores musicales}

La consideración de la música como un lenguaje propio, nos conduce necesariamente a que los valores de los diferentes atributos o rasgos considerados adquieran un valor relacional, por combinación entre ellos. Más allá de su posi- 


\section{Gráfico 3}

\section{Estructura factorial de la emociones}

EMOCIONES

DINÁMICAS

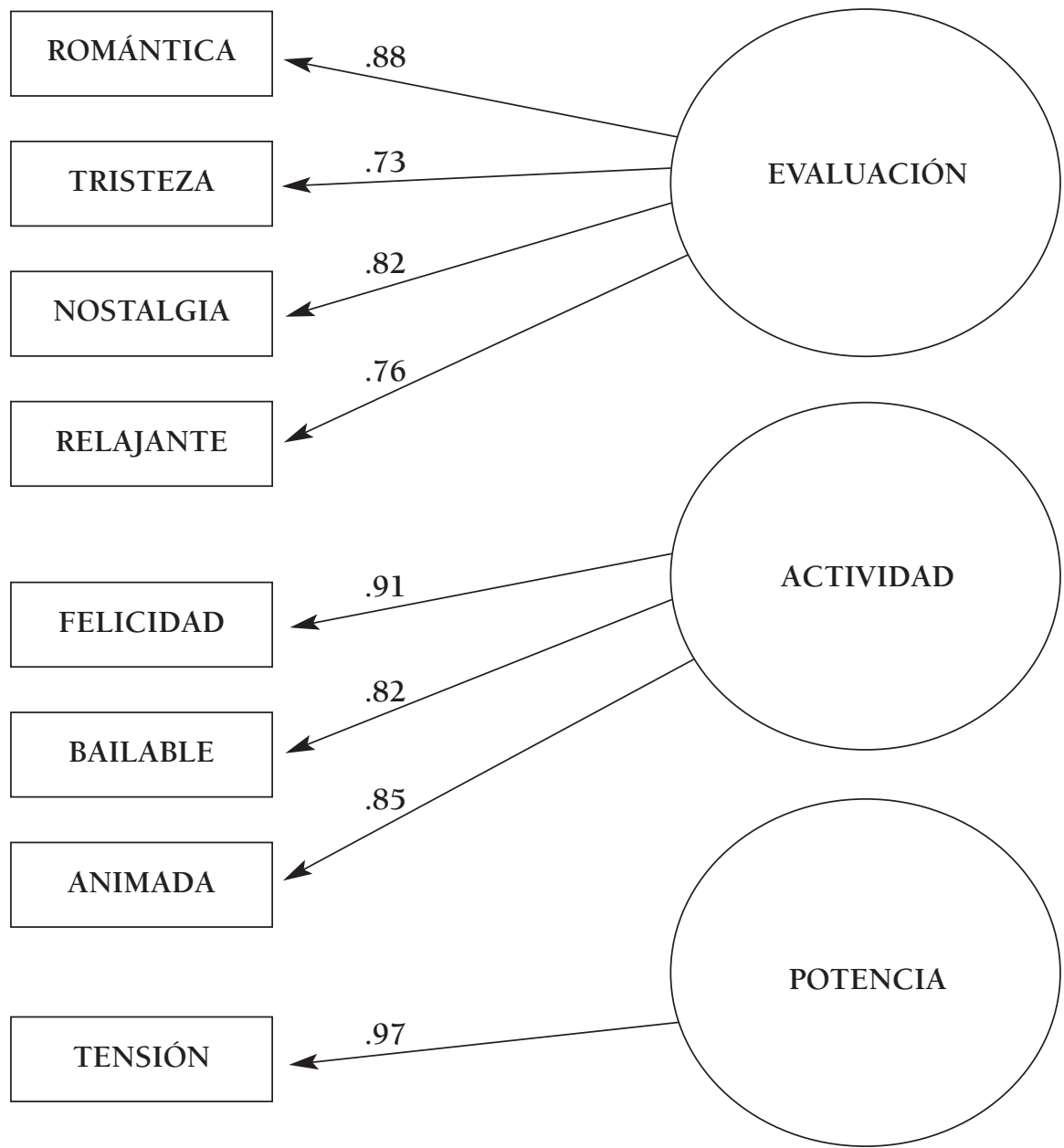

Fuente: elaboración propia

ble valor absoluto teórico. Este valor puede ser estimado empíricamente, en este caso, mediante el análisis de los rasgos considerados y su combinación en las diferentes canciones, cuyas propiedades emocionales hemos determinado.

Para ello, se ha empleado una variante del análisis de componentes principales (Categorical Principal Components Analysis). Este procedimiento esta- 
dístico cuantifica variables categoriales (por ejemplo, nominales) al mismo tiempo que reduce la dimensionalidad de los datos ${ }^{1}$. El escalamiento óptimo permite escalar variables en diferentes niveles de medición. Cada variable categórica es cuantificada de forma óptima en cada una de las dimensiones consideradas. Para ello, partiendo de una atribución nominal, se optimiza la capacidad de agrupamiento en las dimensiones de forma que se atribuye, en este caso, un valor relacional a las diferentes categorías.

\section{Gráfico 4}

Saturaciones en componentes

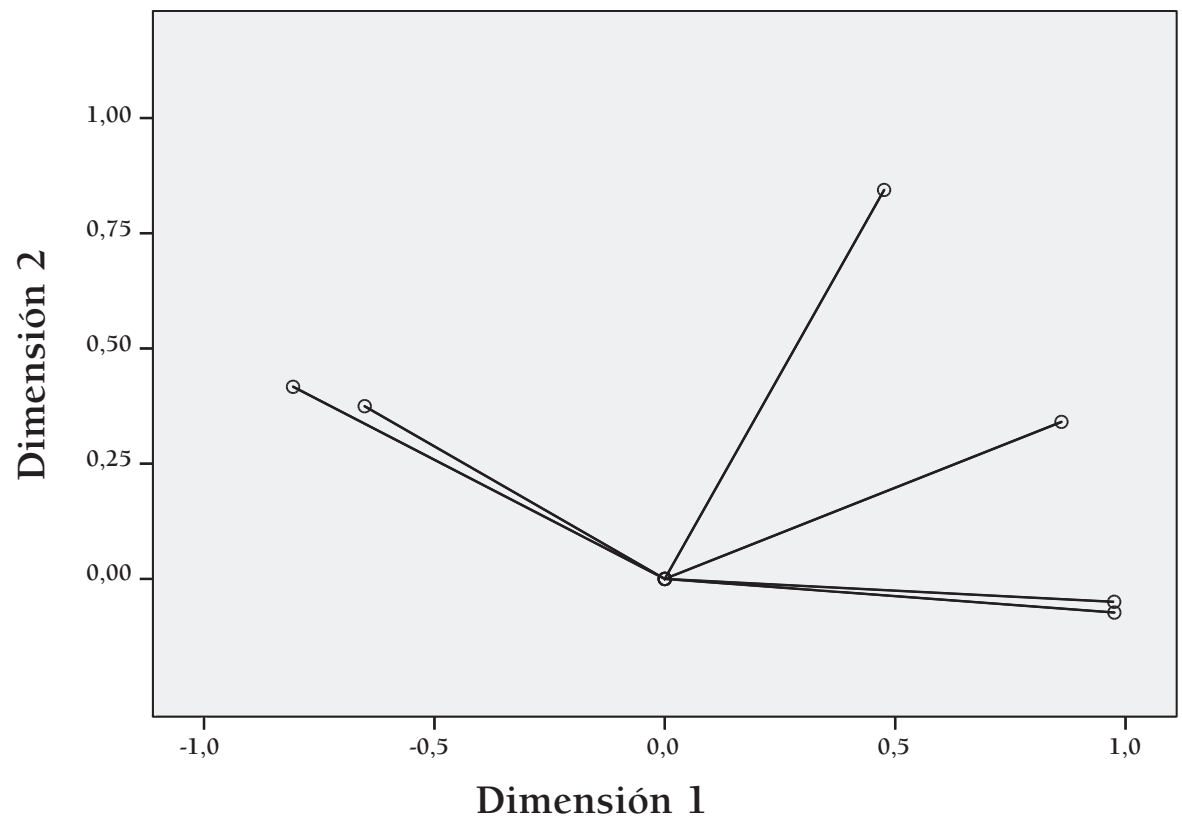

Normalización principal por variable

Fuente: elaboración propia

Dimensionalmente se aprecian dos dimensiones principales, que explican el $84,9 \%$ de la varianza combinada de las cualidades musicales de las canciones consideradas.

${ }^{1}$ En este análisis, para la estimación del escalamiento óptimo en función a la relación entre las categorías que clasifican los rasgos musicales, se empleó el escalamiento óptimo mediante ALS (Mínimos cuadrados alternantes), considerando que algunas de las variables no son nominales múltiples, y se usa un único conjunto de datos. Se empleó el método de asignación de rangos, normalización principal por variable, con criterios de convergencia en ,00001 y un máximo de 100 iteraciones. Sin tratamiento de casos perdidos al no existir en las bases de datos. 
Tabla 2

\begin{tabular}{|c|c|c|c|}
\hline \multicolumn{4}{|c|}{ Resumen del modelo } \\
\hline \multirow[t]{2}{*}{ Dimensión } & Alfa de Cronbach & \multicolumn{2}{|c|}{ Varianza explicada } \\
\hline & & Total (Autovalores) & $\%$ de la varianza \\
\hline 1 & ,897 & 3,947 & 65,785 \\
\hline 2 &, 148 & 1,150 & 19,169 \\
\hline Total & $965^{\mathrm{a}}$ & 5,097 & 84,954 \\
\hline
\end{tabular}

a. El Alfa de Cronbach Total está basado en los autovalores totales.

Fuente: elaboración propia

Los resultados del escalamiento óptimo son los siguientes, comparados con los valores originales de los datos.

Tabla 3. Volumen Cuantificación

\begin{tabular}{lr|r|r|r}
\hline & & \multicolumn{2}{|c|}{ Volumen Cuantificación } & \multicolumn{2}{c}{ Total } \\
\cline { 3 - 5 } & & $-1,00$ & 1,00 & \\
\hline Volumen & DÉBIL & 108 & 0 & 108 \\
\hline \multirow{2}{*}{ Total } & FUERTE & 0 & 108 & 108 \\
& & 108 & 108 & 216 \\
\hline
\end{tabular}

Fuente: elaboración propia

Tabla 4. Tensión Cuantificación

\begin{tabular}{ll|r|r|r}
\hline & & \multicolumn{2}{|c|}{ Tensión Cuantificación } & \multicolumn{2}{c}{ Total } \\
\cline { 3 - 5 } & &,- 77 & 1,29 & \\
\hline Tensión & NO & 135 & 0 & 135 \\
\hline \multirow{2}{*}{ SÍ } & 0 & 81 & 81 \\
Total & & 135 & 81 & 216 \\
\hline
\end{tabular}

Fuente: elaboración propia 
Tabla 5. Tonalidad Cuantificación

\begin{tabular}{|c|c|c|c|c|c|}
\hline & \multicolumn{3}{|c|}{ Tonalidad Cuantificación } & \multirow[t]{2}{*}{ Total } \\
\hline & & $-1,23$ &, 43 & 1,20 & \\
\hline \multirow[t]{2}{*}{ Tonalidad } & MENOR & 0 & 0 & 54 & 54 \\
\hline & MAYOR & 81 & 0 & 0 & 81 \\
\hline & $\begin{array}{r}\text { MENOR Y } \\
\text { MAYOR }\end{array}$ & 0 & 81 & 0 & 81 \\
\hline Total & & 81 & 81 & 54 & 216 \\
\hline
\end{tabular}

Fuente: elaboración propia

Tabla 6. Textura Cuantificación

\begin{tabular}{|c|c|c|c|c|}
\hline & \multicolumn{2}{|c|}{ Textura Cuantificación } & \multirow{2}{*}{ Total } \\
\hline & &,- 58 & 1,73 & \\
\hline Textura & COMPLEJO & 162 & 0 & 162 \\
\hline & SIMPLE & 0 & 54 & 54 \\
\hline Total & & 162 & 54 & 216 \\
\hline
\end{tabular}

Fuente: elaboración propia

Tabla 7. Voz Cuantificación

\begin{tabular}{|c|c|c|c|c|c|}
\hline & \multicolumn{3}{|c|}{ Voz Cuantificación } & \multirow[t]{2}{*}{ Total } \\
\hline & &,- 74 &, 53 & 1,58 & \\
\hline \multirow[t]{3}{*}{$\overline{\mathrm{Voz}}$} & FEMENINO & 0 & 27 & 0 & 27 \\
\hline & MASCULINO & 135 & 0 & 0 & 135 \\
\hline & MIXTO & 0 & 0 & 54 & 54 \\
\hline Tota & & 135 & 27 & 54 & 216 \\
\hline
\end{tabular}

Fuente: elaboración propia

Tabla 8. Tiempo Cuantificación

\begin{tabular}{|c|c|c|c|c|}
\hline & \multicolumn{3}{|c|}{ Tiempo Cuantificación } & \multirow[t]{2}{*}{ Total } \\
\hline & $-1,00$ & ,94 & 1,16 & \\
\hline LENTO & 108 & 0 & 0 & 108 \\
\hline RÁPIDO & 0 & 0 & 27 & 27 \\
\hline RÁPIDO Y LENTO & 0 & 81 & 0 & 81 \\
\hline Total & 108 & 81 & 27 & 216 \\
\hline
\end{tabular}

Fuente: elaboración propia 
El gráfico 5 nos muestra la relación, partiendo del grupo de canciones consideradas, de las cualidades con las dos dimensiones estimadas empíricamente. Debe advertirse que será preciso más estudios posteriores, ampliando la muestra de canciones y de emociones, de forma que la dimensionalidad observada en las cualidades musicales se confirmen o no, como propia de cada una de las emociones. Se han estimado dos dimensiones, aún sin "nombre" y denominadas Dimensión 1 y Dimensión 2. La Dimensión 2 afecta sobre todo a la Voz y a la Tonalidad. La Dimensión 1 influye en mayor grado (varianza explicada) y a un mayor número de cualidades. Solamente la tonalidad no posee carga significativa en la dimensión 1.

\section{Gráfico 5}

DIMENSIÓN

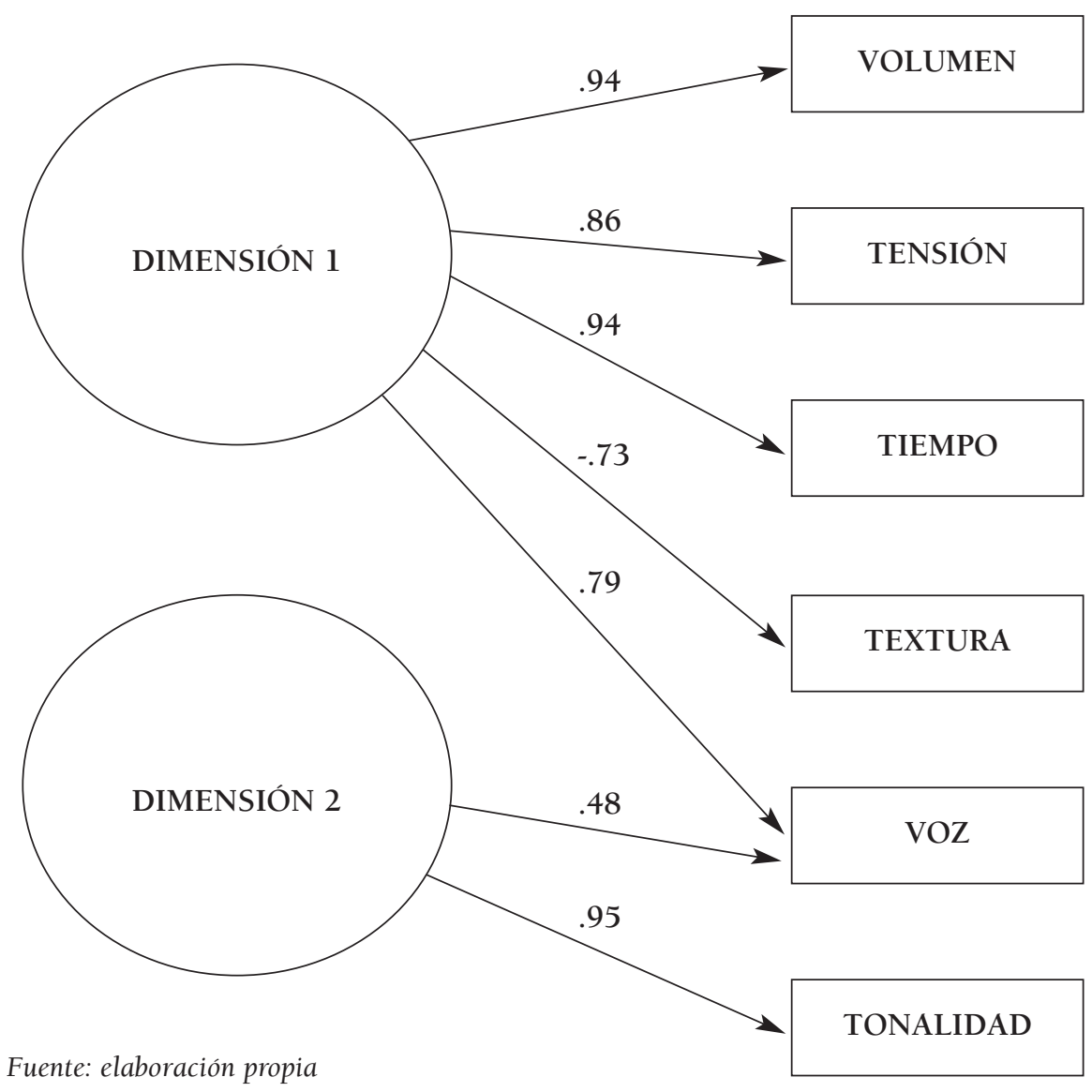


El análisis de la dimensionalidad en las cualidades musicales ya apunta a una relación implícita con los sentimientos que se asocian a las canciones consideradas. Así, graficadas las puntuaciones de las canciones según las emociones que se le asocian en el espacio definido por las dimensiones de las cualidades musicales, se aprecia la estructura factorial siguiente (gráfico 6). La felicidad aparece bastante próxima a la tensión, en la medida que como comentábamos anteriormente, se interpreta en parte como "Actividad" o animación, más que como un estado de ánimo particular.

\section{Gráfico 6}

Puntos de objeto etiquetados mediante Números de caso

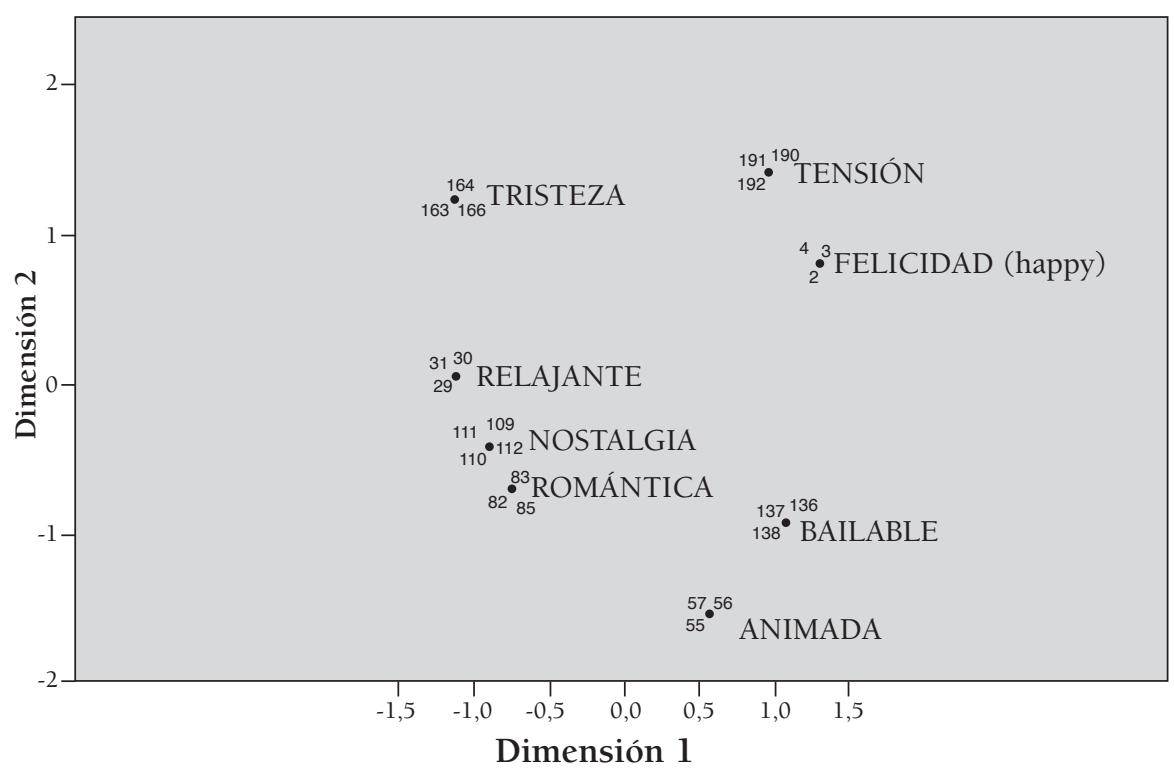

Normalización principal por variable

Fuente: elaboración propia

Por último consideremos la relación empírica entre las dimensiones de diferencial semántico (Evaluación, Potencia y Actividad), y las dimensiones de las cualidades musicales que muestran las canciones consideradas. Estos son coeficientes estandarizados (beta) que permiten comparar la importancia de cada dimensión (rasgos musicales) en las dimensiones de diferencial semántico.

Actividad $=.574$ Dimensión $1+-.398$ Dimensión 2
$\mathrm{t}$
$(11,722)$
$(-8,123)$
$\mathrm{R}^{2}=, 488$ 


$$
\begin{array}{llll}
\text { Potencia }= & .164 \text { Dimensión } 1+ & & \\
\mathrm{t} & (2,558) & (4,958) & \text { Dimensión } 2 \\
& \text { Evaluación }=-.713 \text { Dimensión } 1 & \mathrm{R}^{2}=, 127 \\
\mathrm{t} & (-14,925) & \mathrm{R}^{2}=, 514
\end{array}
$$

En el caso de la actividad (como variable latente asociada a los estados de ánimo) la Dimensión 1 muestra un mayor efecto (.574), con carácter positivo, incrementando la sensación de actividad. La dimensión 2 tiene comparativamente un efecto menor sobre la percepción de Actividad (-.398), siendo además de carácter negativo. En el caso de la Potencia, el mayor efecto procede de la Dimensión 2 (.317), siendo, también positivo el inducido por la Dimensión 1 (.164). Por último, en la Evaluación parece influir de forma estadísticamente significativa solamente la Dimensión 1 (.-713). Debe considerarse estas relaciones como unas conclusiones provisionales, tanto en la especificación de la estructura como en la magnitud de los coeficientes. Será preciso efectuar más estudios de validación que permitan conocer las estructuras anteriores en toda su extensión.

\subsection{Las emociones y el lenguaje musical}

Este análisis completa el diseño, integrando las dos grandes áreas de interés: las emociones desde la medición empírica de los sujetos y las cualidades musicales de las canciones en estudio. Su relación estadísticamente significativa y teóricamente sustantiva es un avance importante que permite habilitar nuevas vías de vinculación entre música y lenguaje. Y no solo en términos emocionales.

El gráfico 7 (pág. 38) muestra la estructura dimensional completa, como modelo estructural y de medición. Los coeficientes han sido estimados empíricamente a partir de las dos muestras obtenidas. Todos ellos son significativos al 0.01 pudiendo rechazarse la posibilidad que el cero este comprendido en el intervalo de confianza del estimado de asociación.

Podemos apreciar como la dimensión de Evaluación viene expresada mediante los estados de ánimo "Romántica", "Tristeza", "Nostalgia" y "Relajante". Esta dimensión se explica y viene generada principalmente por la Dimensión 1 (-.71) que se expresa mediante los rasgos musicales de "Volumen", "Tensión", "Tiempo", "Textura" y "Voz". La Dimensión 1 explica también las dimensiones de Actividad (.57) y de Potencia (-.39). Claramente su influencia es menos especializada que en el caso de la Dimensión 2. Esta dimensión expresa rasgos como la "Voz" y la "Tonalidad". La Dimensión 2 influye en la percepción de Actividad (.16) definida mediante los indicadores de "Felicidad", "Bailable" y "Animada", y de Potencia (.31), reflejada en la producción de "Tensión". 


\section{Gráfico 7}

Modelo estructural de medición: rasgos musicales y emociones

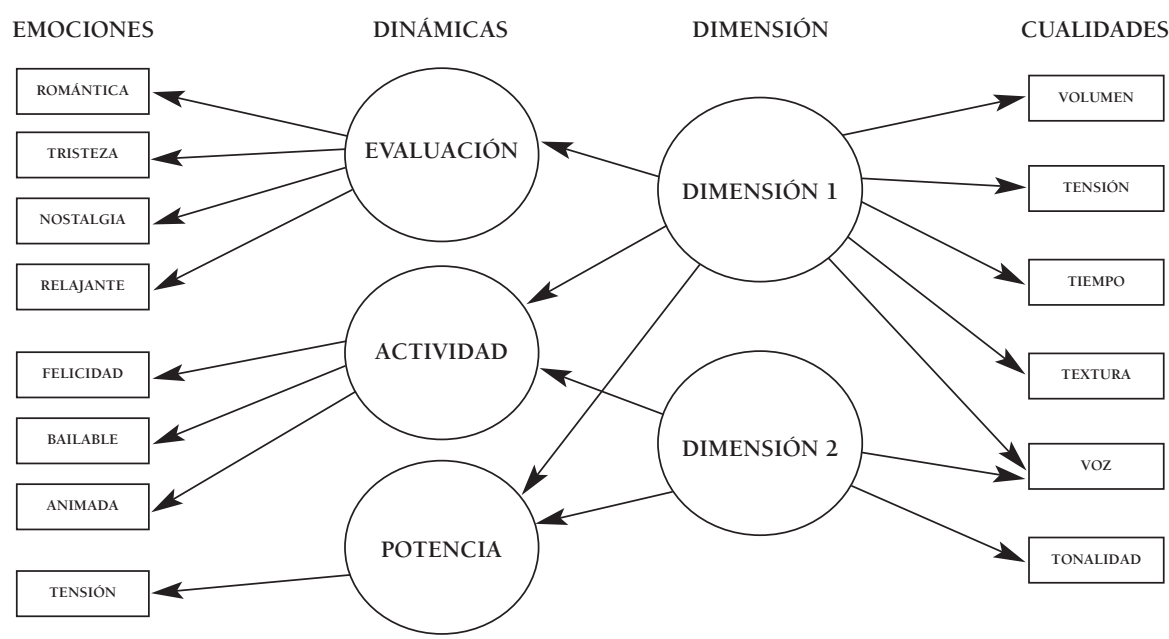

Fuente: elaboración propia

En ese sentido existe una relación empírica entre los rasgos musicales y las emociones, medidas estas en su dimensionalidad como diferencial semántico. Parece evidente que la articulación entre los dos planos, las emociones producidas por la música y los rasgos del lenguaje musical, se establece en términos de variables latentes. La relación entre la diversidad de emociones consideradas y los elementos de expresión musical, se establece canalizada por variables latentes que controlan las diferentes combinaciones y sus efectos.

\section{CONCLUSIONES}

El estudio de la influencia de la música en la generación de estados emocionales es un área que experimenta en la actualidad un gran dinamismo. Tanto en la forma de investigación fundamental, orientada a un mejor conocimiento de los procesos cognitivos, desde las neurociencias y la psicoacústica, como aplicada, por ejemplo a la optimización de procesos comunicacionales. Entre las conclusiones cabe señalar que 1) Existe una relación estadística significativa entre la asociación de sentimientos a las canciones propuestas, primero "motu propio" (espontáneamente), y después validada externamente por otros sujetos entrevistados. 2) Existe dimensionalidad en la estructura de valoración de las canciones, que corresponde con las propuestas de dimensionalidad en la orientación psicológica del diferencial semántico. 3) Existe una relación empí- 
rica estadísticamente significativa entre las cualidades musicales de las canciones y su efecto psicológico tal y como lo reconocen los entrevistados. El campo de investigación es claramente interdisciplinar, incorporando conocimientos y competencias en música, escalamientos psicométricos, análisis estadístico, psicoacústica o comunicación. Otras disciplinas como la neurofisiología son especialmente activas en el estudio de los procesos cognitivos de carácter neuronal.

En resumen la presente investigación aporta evidencias para la validación de la conexión entre música y emociones, utilizando datos empíricos mediante un diseño test-retest. Valida la importancia del fenómeno musical en la producción de emociones y que, en el caso de las canciones, actúa de forma independiente a los contenidos semánticos, es decir de las letras de las canciones. Se aprecian efectos de canciones cuyas letras están en inglés y otros idiomas en la que los sujetos carecen de competencia lingüística. La música muestra rasgos fundamentales, desde el análisis de lenguaje musical, que se asocian a la presencia de determinadas cualidades psicológicas. Aporta la evaluación de los componentes dimensionales presentes en las diferentes emociones consideradas. Facilita nuevas evidencias sobre la dimensionalidad en la orientación psicológica básica hacia conceptos "musicales" (canciones), partiendo de las propuestas clasificatorias de los propios individuos (jóvenes) considerados. Así mismo, contribuye con un diseño de investigación innovador en el campo de estudio, dado que la estrategia habitual es facilitar a los sujetos diferentes músicas que han sido preclasificadas por el investigador. (Vieillard et alt; 2008). En esta investigación se parte de una fase exploratoria donde los sujetos aportan la relación estado emocional y música (EMIC), posteriormente se analiza los elementos musicales que contienen las canciones (ETIC) para determinar sus rasgos característicos. Finalmente, se valida con una segunda muestra donde se pide que se valore la presencia de los componentes básicos considerados (EMIC). En ese sentido, profundizar en el análisis permitirá desarrollos en el estudio del ajuste entre mensajes y elementos musicales, con la finalidad de optimizar la comunicación, por ejemplo, en disciplinas como la publicidad. Aporta elementos de herramienta para mejorar la compatibilidad en la eficacia performativa de los dos lenguajes: musical y lenguaje natural con la finalidad de aunar significados y emociones. En cierto modo, este estudio anticipa una propuesta en la programación de rutinas de diagnóstico que permitan efectuar un primer análisis clasificatorio estándar de la potencialidad emocional de las canciones.

Por último, una aportación prometedora es la conexión entre las dimensiones de diferencial semántico y las cualidades de la música. Permite abrir una 
línea con gran potencial al poner en relación empírica, gracias a los efectos psicológicos, ambos lenguajes (natural y musical). La investigación aporta datos empíricos, estrategias de medición y escalamiento así como conclusiones que desde el punto de vista aplicado, representa las bases para el desarrollo de software de análisis y diagnóstico de la eficacia emocional con posibilidad de empleo en múltiples ámbitos, especialmente de comunicación y publicidad.

\section{BIBLIOGRAFÍA}

Alaminos, A.F. (2014) Cuando las letras son música: las canciones en inglés en la publicidad española. Universidad de Alicante.

Alaminos, A.F. y Santacreu, O. (2004) "Let the music play the feelings. The performative effect of music in advertising". Proceedings of the Conference on Interdisciplinary Musicology. Vienna.

Aristoteles (1982). Política. 10 edición. México: Porrúa.

Blood, A.J., Zatorre, R.J., Bermudez P. \& Evans, A.C. (1999). Emotional responses to pleasant and unpleasant music correlate with activity in paralimbic brain regions. Nat. Neurosci. 2: 382-387.

Blood, A. \& Zatorre R.J. 2001. Intensely pleasurable responses to music correlate with activity in brain regions implicated in reward and emotion. Proc. Natl. Acad. Sci. 98: 11818-11823.

Brown, S., Martinez, M. \& Parsons, L.M. (2004). Passive music listening spontaneously engages limbic and paralimbic systems. Neuroreport 15: 2033-2037.

Fritz, T. Sebastian Jentschke, S. Gosselin, N. Sammler, D. Peretz, I. Turner, R. Friederici A.D. and Koelsch, S. (2009) "Universal Recognition of Three Basic Emotions in Music". Current Biology, Volume 19, Issue 7, 573-576, 19 March

Hannon E. and Trainor L. (2007) Music acquisition: effects of enculturation and formal training on development. Trends in Cognitive Sciences, Volume 11, Issue 11, 466-472, 1 November

Juslin Patrick N. and Sloboda John A. (eds.) (2010) Music and emotion. Theory, research, applications. Oxford: Oxford University Press.

Koelsch, S., Gunter, T.C., Friederici A.D. y Schröeger E. (2000). Brain indices of music processing: "Nonmusicians" are musical. J. Cogn. Neurosci. 12: 520-541.

Koelsch, S. (2005) "Investigating Emotion with Music. Neuroscientific Approaches." Annals New York Academy of Sciences. 1060: 1-7.

Koelsch, S. y Friederico A.D. (2003). Toward the neural basis of processing structure in music: comparative results of different neurophysiological investigation methods. Ann. N. Y. Acad. Sci. 999: 15-28.

Koelsch S, Kasper E, Sammler D, Schulze K, Gunter T, et al. (2004) "Music, language and meaning: Brain signatures of semantic processing". Nat Neurosci 7: 302307. 
Koelsch S. and Siebel, W. (2005). "Towards a neural basis of music perception" Trends in Cognitive Sciences, Volume 9, Issue 12, 578-584, December

Koelsch S, Fritz T, von Cramon DY, Müller K, Friederici AD (2006) Investigating emotion with music: An fMRI study. Hum Brain Mapp 27: 239-250.

Koelsch S. (2010) "Towards a neural basis of music-evoked emotions" Trends in Cognitive Sciences, Volume 14, Issue 3, 131-137, 11 February 2010.

Konecni Vladimir J. (2010) "The influence of affect on music choice". Juslin Patrick N. and Sloboda John A. (eds.) Music and emotion. Theory, research, applications. Oxford: Osford University Press. Pags. 697-723

Krumhansl, C.L. (1997). An exploratory study of musical emotions and psychophysiology. Can. J. Exp. Psychol. 51: 336-353.

Levitin, D.J. \& Menon, V. (2003). Musical structure is processed in "language" areas of the brain: a possible role for Brodmann Area 47 in temporal coherence. Neuroimage 20: 2142-2152.

Mitterschiffthaler, M.T., Fu, C.H.Y., Dalton, J.A., Christopher M. Andrew, C.M.,Williams, S.C.R. "A functional MRI study of happy and sad affective states induced by classical music". Human Brain Mapping Volume 28, Issue 11, pages 1150-1162, November 2007

Miranda, R.A., Ullman, M.T. (2007) Double dissociation between rules and memory in music: An event-related potential study. Neuroimage 38: 331-345

Menon, V. \& Levitin, D.J. (2005). The rewards of music listening: response and physiological connectivity of the mesolimbic system. Neuroimage 28: 175-184.

Meyer, L.B. (1956). Emotion and meaning in music. University of Chicago Press. Chicago.

North A.C. and Hargreaves, D.J. "Music and Marketting" en Juslin Patrick N. and Sloboda John A. (eds.) (2010) Music and emotion. Theory, research, applications. Oxford: Osford University Press. pags. 909-932.

Rowel, L. (1983) Introducción a la filosofía de la música. Edisa. Barcelona

Santacreu, O. (2002) La música en la publicidad. Universidad de Alicante. Alicante

Seguí, S. (1991) Teoría Musical (II). Ed. Unión musical. Madrid.

Sloboda, J.A. 1991. Music structure and emotional response: some empirical findings. Psychol. Music 19: 110-120.

Steinbeis N, Koelsch S (2008) "Comparing the Processing of Music and Language Meaning Using EEG and fMRI Provides Evidence for Similar and Distinct Neural Representations". PLoS ONE 3(5): e2226. doi:10.1371/journal.pone.0002226

Vieillard, S. Peretz, I. Gosselin, N. Stéphanie Khalfa, S. Lise Gagnon y Bernard Bouchard; (2008) "Happy, sad, scary and peaceful musical excerpts for research on emotions" Cognition \& Emotion Volume 22, Issue 4, pages 720-752

Zatorre, R. Chen, J. \& Penhune, V. (2007) "When the brain plays music: auditory-motor interactions in music perception and production" Nature Reviews Neuroscience 8, 547-558 (July 2007) 
Zentner, M.; Grandjean, D.; Scherer, K. R. (2008) "Emotions evoked by the sound of music: Characterization, classification, and measurement." Emotion, Vol 8(4), Aug, 494-521

ANTONIO FRANCISCO ALAMINOS FERNÁNDEZ está especializado en el estudio de las capacidades performativas de la música. Título profesional en violín, cursa en la actualidad estudios profesionales de viola. Es Bachelor en Circumpolar Studies por la Universidad de Nordland (Noruega), y Grado en Publicidad y Relaciones Públicas por la Universidad de Alicante (España).

Recibido: 12/01/2014 Aceptado: 07/05/2014 